\title{
« JE EST UN AUTRE ». LE PASTICHE ET LA PARODIE CHEZ PIERRE LOUŸS
}

\author{
Vendula ŠTÁFOVÁ \\ Chercheur indépendant
}

\begin{abstract}
En): The paper deals with the work of the French poet and writer, dandy and libertine, Pierre Louÿs (1870-1925) who devoted his entire life to erotic and pornographic writings. Throughout his life, Louÿs took a particular and very intense pleasure in pastiching or parodying the classical texts of French literature. Therefore, a number of stylistic exercises imitating the writing style of many great French writers emerged in Louÿs' work. Besides, one of his favourite activities was to sign his own pornographic poems with the names of his literary colleagues. It should be noted that even Louÿs' own literal work represents a kind of pastiche. The famous Songs of Bilitis (Les Chansons de Bilitis, 1894) were published as the lost works of a disciple of Sappho. What made them sensational is the fact that Louÿs claimed that the poems were the work of an ancient Greek courtesan and contemporary of Sappho, Bilitis; while to himself, Louÿs ascribed the modest role of a mere translator. The Woman and the Puppet (La Femme et le Pantin, 1898) is in fact the transposition of one episode from the life of Giacomo Casanova and there is also an evident inspiration by Carmen by Prosper Merimée. The pornographic Island of Women (L'Ille aux dames, 1988) is a parody of The Mysterious Island by Jules Verne and at the same time a parody of The Adventures of King Pausole (Les Aventures du roi Pausole, 1901). Furthermore, all Louÿs' writings are heavily eroticised, sometimes even verging on pornography. For example, The Young Girl's Handbook of Good Manners for Use in Educational Establishments (Manuel de Civilité pour les petites filles à l'usage des maisons d'éducation, 1926) remains his most outrageous as well as a truly hilarious piece. As a matter of fact, the comic is an inseparable element of Louÿs' writings. Undoubtedly, this keen sense of the comic adds to the writings of Pierre Louÿs a character of originality, allowing him to create real masterpieces of the genre.
\end{abstract}

Keywords (En): Pierre Louÿs ; Fin de siècle ; pastiche ; parody ; literary mystification ; erotic literature ; pornographic literature; humor

Mots-clés (Fr): Pierre Louÿs ; Fin de siècle ; pastiche ; parodie ; mystification littéraire ; littérature érotique ; littérature pornographique ; humour

Pendant toute sa vie, l'écrivain français fin-de-siècle Pierre Louÿs fut fidèle à ses trois lubies : le papier blanc, les vieux livres et les femmes brunes. Ces trois caprices pourraient d'ailleurs suffire à le définir comme un auteur qui passait son temps à écrire inlassablement sur les femmes tout en rendant hommage à ses précurseurs littéraires.

Il est vrai qu'on remarque dans l'écriture louÿsienne une grande dépendance à l'égard des textes antérieurs, tels que Gérard Genette les a dressés dans ses Palimpsestes (1982). Cette relation intertextuelle entre l'hypotexte et l'hypertexte louÿsien est parfois discrète mais très souvent flagrante. Il paraît même que l'écrivain ne se passait pas d'un point de départ dans l'œuvre hypotextuelle pour déclencher son imagination littéraire. Le plus souvent, c'est dans l'œuvre érotique de Louÿs, aussi vaste que scandaleuse, qu'on trouve non seulement l'inspiration et la transposition des œuvres littéraires antérieures mais également leur détournement parodique. 
Tel est par exemple le cas des Douze douzains de dialogues ou Petites scènes amoureuses (1927), souvent rééditées comme Dialogues de courtisanes, qui sont fortement influencés par les Scènes de la vie des courtisanes de Lucien dont Louÿs publia la traduction en 1894. C'est également la lecture de Lucien qui inaugurera chez l'écrivain ce qu'on pourrait appeler le cycle louÿsien de courtisanes. Si une courtisane tient toujours le rôle principal dans Les Chansons de Bilitis (1895), Aphrodite, mours antiques (1896) et La Femme et le Pantin (1898), on en retrouve également une dans Les Aventures du roi Pausole (1901), dans L'homme de pourpre (1901) ou dans un roman inachevé intitulé Morphise. En outre, la lecture attentive de Lucien donna naissance au fabuleux livre mystificateur des Chansons de Bilitis (1895) que Louÿs a préparé pendant plusieurs années.

Pour ses Chansons de Bilitis, l'écrivain n'a rien laissé au hasard et il s'y est soigneusement préparé, ayant écrit deux mille pages de brouillons. Finalement, en 1895, Les Chansons de Bilitis, traduites du grec pour la première fois par un certain « $P$. $L$. », voient le jour. Louÿs fait précéder sa prétendue traduction d'une sorte de préface résumant la vie de Bilitis et expliquant la découverte de sa poésie dans sa sépulture. On apprend donc que le tombeau de Bilitis, tout comme ses poésies, gravées dans la pierre, avaient été récemment retrouvés à Chypre par un respectable archéologue allemand, le professeur G. Heim et que l'écrivain s'est servi de ses travaux pour sa traduction française. ${ }^{1}$

Après la parution du recueil, Louÿs guettait impatiemment la réaction des plus éminents hellénistes et philologues de l'époque. Leurs réponses ne se firent pas attendre et l'une d'elles plongea l'écrivain dans une hilarité féroce : Gustave Fougère, professeur d'archéologie grecque à la faculté de Lille, le remerciait d'avoir si bien réussi la traduction du texte grec.

Or, ce n'est pas uniquement grâce aux formules archaïsantes et à une excellente connaissance de la culture antique que Louÿs a réussi son pastiche de la poésie grecque. S'il est parvenu à imiter le style de la poésie hellène si parfaitement que personne n'a douté de l'authenticité de l'œuvre, c'est parce qu'il a projeté dans son écriture une vraie énonciation féminine. Autrement dit, Les Chansons de Bilitis représentent un discours poétique dans lequel le «je» masculin traditionnel ne s'adresse plus à un «tu » féminin, mais le locuteur et le personnage central apparaissent comme féminins. La féminité des poèmes est donc assurée par une forte présence de figures féminines, plus exactement par le caractère féminin de l'écriture entière :

Hélas, si je pense à elle, ma gorge se dessèche, ma tête retombe, mes seins durcissent et me font mal, je frissonne et je pleure en marchant. Si je la vois, mon cœur s'arrête, mes mains tremblent, mes pieds se glacent, une rougeur de feu monte à mes joues, mes tempes battent douloureusement. Si je la touche, je deviens folle, mes bras se raidissent, mes genoux défaillent. Je tombe devant elle, et je me couche comme une femme qui va mourir. De tout ce qu'elle me dit je me sens blessée. Son amour est une torture et les passants entendent mes plaintes... Hélas ! Comment puis-je l'appeler Bien-aimée ? (LOUŸs, 1895 : 91)

\footnotetext{
${ }^{1}$ Évidemment, Herr Professor G. Heim n'avait jamais existé ; son nom (en allemand geheim) signifie tout simplement secret.
} 
Les Chansons de Bilitis vaudront au Louÿs mystificateur plusieurs années de distraction. Plus tard, il interviendra même dans la polémique sur l'authenticité et l'originalité du recueil avec un texte astucieux intitulé Bilitis a-t-elle existé ? qui incita le public à de nouveaux débats. Quant à Bilitis, elle continuait de figurer dans la littérature savante (par exemple dans la Bibliographie pratique de la littérature grecque des origines à la fin de la période Romaine de Paul Masqueray de 1914 qui prenait la «traduction » de Louÿs pour un travail médiocre).

Évidemment, ce genre de plaisanterie convenait tout à fait au caractère malicieux de l'écrivain. Pourtant, sa réputation de mystificateur voulant se divertir aux dépens de ses contemporains se retourna contre lui, lorsqu'il voulut présenter ses recherches sérieuses sur Corneille et Molière. Cette fois-ci, personne ne prit son travail au sérieux.

La lubie de Louÿs consistant à tromper le public se mariait avec un caprice malicieux de se moquer de lui à chaque occasion. À titre d'exemple, l'une de ses activités favorites consistait à signer de noms célèbres ses propres poèmes pornographiques. Le grave poète philosophique Sully Prudhomme, premier lauréat du prix Nobel de littérature, serait sûrement surpris de lire ce petit poème badin tracé sur une carte postale représentant deux jeunes filles couchées dans le même lit :

\footnotetext{
Admirez les gestes paisibles

Des vierges aux cheveux coupés

Leurs doigts sont peut-être invisibles

mais ne sont pas inoccupés...

J'ignore ce qu'il vous en semble

Mais les parents sont hasardeux

De laisser deux filles ensemble

Sans me coucher entre les deux
}

Sully Prudhomme (Goujon, 2012 : 34)

Le comique est un élément inséparable de l'écriture louÿsienne, notamment de son écriture érotique car il est vrai que Louÿs savait introduire dans son écriture une bonne dose d'humour. Nous partageons le point de vue de Jean-Paul Goujon, spécialiste de l'œuvre louÿsienne ainsi que de la littérature libertine, qui écrit à ce propos qu'il y avait chez Louÿs «un don inné pour voir le côté comique ou ridicule des gens, des choses et des situations. De là sa passion pour la parodie, et spécialement la parodie érotique. » (GoUJON, 2012 : 34)

L'étonnant mimétisme de l'écrivain lui fit composer un grand nombre de textes variés qui se distinguent par un érotisme exalté ainsi que par un ton railleur. D'ailleurs, pendant toute sa vie, il éprouvait un plaisir particulier et très intense à pasticher ou à parodier des textes classiques réputés éminemment respectables. Il existe tout un recueil publié en 1981 sous le titre de Pastiches et Parodies qui rassemble des poèmes libres de Louÿs imitant des textes célèbres de La Fontaine, Hugo, Daudet, Bruant, Musset et bien d'autres en leur donnant une connotation grivoise. L'exemple de La Nuit de mai d'Alphonse de Musset, ainsi 


\section{métamorphosé par la fantaisie de Louÿs, nous semble être à cet égard assez} éloquent :

Alphonse de Musset : La Nuit de Mai

La Muse.

Poëte, prends ton luth et me donne un baiser ;

La fleur de l'églantier sent ses bourgeons éclore.

Le printemps naît ce soir ; les vents vont s'embraser ;

Et la bergeronnette, en attendant l'aurore,

Aux premiers buissons verts commence à se poser.

Poëte, prends ton luth, et me donne un baiser.

Le Poëte.

Comme il fait noir dans la vallée !

J'ai cru qu'une forme voilée

Flottait là-bas sur la forêt.

Elle sortait de la prairie ;

Son pied rasait l'herbe fleurie ;

C'est une étrange rêverie ;

Elle s'efface et disparaît.

La Muse.

Poëte, prends ton luth ; la nuit, sur la pelouse, Balance le zéphyr dans son voile odorant.

La rose, vierge encor, se referme jalouse

Sur le frelon nacré qu'elle enivre en mourant.

Écoute ! tout se tait ; songe à ta bien-aimée.

Ce soir, sous les tilleuls, à la sombre ramée

Le rayon du couchant laisse un adieu plus doux.

(De Musset, 1888 : 98-99)

Pierre Louÿs : La Nuit d'Avril

La Muse

Poète, prend mon rut, j'ai besoin de baiser.

La fleur de mes poils noirs s'entrouvre, près d'éclore.

Le printemps naît ce soir, les cons vont s'embrasser

Et le doigt de la vierge en attendant l'aurore

Sur la motte en chaleur commence à se poser.

Poète, prend mon rut, j'ai besoin de baiser.

Le Poète

(en regardant sous la tunique de la Muse)

Comme il fait noir dans ta vallée!

J'ai cru qu'une chair mal branlée

Brillait là-bas sous ta forêt.

Elle sortait de la prairie...

C'est ton clitoris en furie

Qui bande avec ta rêverie, 
Est-ce bien lui qui m'apparaît?

La Muse

Poète, prend mon rut; la Nuit sur la pelouse

Berce un godemiché bleu dans son voile odorant.

La vulve, triste encor, se referme jalouse

Sur le vit inconnu qu'elle enivre en pleurant.

Écoute: on fout partout. Songe à ta bien-aimée.

Ce soir sous les tilleuls, tu la laissas pâmée

Avec un lac de sperme entre les deux genoux.

(GOUJON, 2012 : 601-602)

L'immense culture de Louÿs, qui dépassait de très loin ce qu'on appelle l'érudition, impliquait nécessairement un tel jeu avec des textes. C'est tout naturellement que l'écrivain se livrait d'abord au pastiche, qui était à ses yeux la preuve d'une excellente connaissance de l'œuvre ainsi que la marque d'une admiration respectueuse. Pourtant, son caractère lui faisait exagérer ses pastiches, qui dérivaient ainsi vers la parodie érotique.

Il est à noter que, tout au long de sa vie, Louÿs, ce pasticheur acharné, doublait systématiquement ses œuvres officielles d'une version érotique, disant ce que le double officiel ne pouvait qu'insinuer. Il finit alors par se pasticher lui-même. Ainsi, Les Chansons de Bilitis (1895), qui sont elles-mêmes un pastiche, ont leur version érotique intitulée Les Chansons secrètes de Bilitis (1933), Aphrodite, mours antiques (1896) sa version libre au titre éponyme (1928) et Les Aventures du Roi Pausole (1901), cette « utopie érotique transposée dans le monde moderne »(GOUJON, 2002 : 495) dans laquelle le lecteur se croit parfois chez Diderot ou Voltaire, seront parodiées dans l'Histoire du roi Gonzalve et des Douze princesses (1927).

Parfois, les relations intertextuelles sont encore plus compliquées et c'est pourquoi la même œuvre de la plume louÿsienne paraît fonctionner comme l'hypotexte en même temps que l'hypertexte. Par exemple le roman La Femme et le Pantin (1898), qui est lui-même la transposition d'un épisode de Casanova inspiré de Carmen (1847) de Prosper Mérimée, sera parodié à certains égards dans Trois filles de leur mère (1926) et, pareillement, mais inversement, L'Île aux dames (1988), représente en effet un travestissement burlesque de L'Île mystérieuse (1874) de Jules Verne en même temps que la parodie des Aventures du roi Pausole (1901).

La comparaison de L'Île aux dames avec l'hypotexte vernien, nous permettra d'esquisser les caractéristiques de l'écriture louÿsienne. Les deux livres commencent identiquement par une chute de ballon sur une île inconnue, mais la ressemblance s'arrête là. Tandis que Verne raconte son histoire comme une robinsonnade dans laquelle les naufragés doivent profiter de toutes leurs connaissances et compétences techniques pour survivre sur une île déserte, Louÿs décrit une terre habitée dont les habitants paraissent n'avoir pour unique occupation que l'exercice de leur sexualité. Si l'hypotexte de Verne fourmille de descriptions technologiques, le texte de Louÿs déborde de détails concernant les descriptions des coutumes, traditions et fêtes des autochtones, comme par exemple 
une procession annuelle de lesbiennes, l'orgie annuelle de la cour, les inventions absurdes de la société insulaire comprenant des bancs larges devant les terrasses de cafés pour les actes d'amour ou des coiffeuses spécialisées dans les poils du pubis, etc.

Sans aucun doute, ce livre inachevé constitue l'une des plus audacieuses œuvres érotiques de Louÿs. On peut y voir autant une critique sévère de l'hypocrisie bourgeoise de la Belle Époque, réprimant la sexualité, qu'un rêve extraordinaire qui semble représenter le monde fantasmé de l'écrivain, le monde idéal selon Pierre Louÿs, un monde tout entier gouverné par la sexualité débridée.

Il est vrai que l'œuvre entière de Louÿs représente une véritable attaque contre la morale bourgeoise dont l'écrivain ridiculise les règles à toute occasion. On y coïte n'importe où et avec n'importe qui, peu importe que ce soit à l'église, au vestiaire, aux toilettes ou au lit; avec la bonne, l'amie, les membres de la famille, les filles impubères ou les animaux. Pierre Louÿs, cette «machine à subvertir la morale » (GouJON, 1991: 62) n'a peur de rien, sa plume ironique ne s'impose aucune limite, ne respectant aucun tabou car pédophilie, zoophilie, inceste, saphisme, scatologie et bien d'autres perversions sexuelles y sont au rendez-vous. Tel est par exemple le cas de son Manuel de civilité pour les petites filles à l'usage des maisons d'éducation (1926), une hilarante parodie de la littérature moralisante, dans lequel l'auteur donne des conseils courts et précieux aux jeunes filles qui ne savent pas encore se comporter :

-Quand vous vous êtes servie d'une banane pour vous amuser toute seule ou pour faire jouir la femme de chambre, ne remettez pas la banane dans la jatte sans l'avoir soigneusement essuyée. (GouJon, $2012: 10$ )

-Une jeune fille bien élevée ne pisse pas dans le piano. (Goujon, 2012:16)

-Si vous baisez l'après-midi dans une église de campagne, ne vous lavez pas le cul dans le bénitier. Loin de purifier votre péché, vous l'aggraveriez au contraire. (Goujon, 2012 : 10)

-Ne grimpez pas sur les socles des statues antiques pour vous servir de leurs organes virils. Il ne faut pas toucher aux objets exposés ; ni avec la main ni avec le cul. (Goujon, 2012 : 20)

-Si vous trouverez un monsieur tout nu dans le lit de mademoiselle votre sœur, n'allez pas le dire tout bas à monsieur votre père. La visite n'est pas pour lui. (Goujon, 2012 : 10)

-Tous les soirs, avant de vous branler, faites votre prière à genoux. (Goujon, $2012: 28$ )

L'écrivain n'abandonne pas ce ton burlesque dans $\operatorname{Pybrac}^{2}$ (1927), parodie des quatrains moralisateurs dans laquelle il détourne le genre originel en une fantaisie érotique, tout en gardant la stricte organisation formelle de son modèle. Ainsi, chacun des trois-cent-treize quatrains du recueil commence par un «Je n'aime pas » avant d'être suivi des plus impudiques anecdotes qu'on puisse imaginer :

Je n'aime pas la mère offrant sa fille morte

(Quatorze ans, quatre poils, pucelle, et cætera)

Disant : « Amusez-vous, mais fermez bien la porte.

\footnotetext{
${ }^{2}$ Guy de Faur de Pibrac (1529-1584), magistrat et poète toulousain du XVIe siècle.
} 
Et pinez-la partout, tant que ça vous plaira.

(GOUJON, 2012: 806)

Pybrac, tout comme les érotiques citées plus haut, met en scène les obsessions louÿsiennes: sodomie, inceste, saphisme, pédophilie ainsi que fantasmes scatologiques, zoophiles ou nécrophiles. Lors de la lecture, il semble en effet que son auteur éprouve une fascination incontrôlable non seulement pour l'orchestration de ces perversités dans le texte mais également pour la répétition constante de certains mots obscènes. Certes, on peut y discerner une sorte de graphomanie, une obsession verbale, mais leur effet est garanti : le rythme répétitif des quatrains provoque au bout du compte un effet quasi hypnotique et le texte se transforme en véritables mantras pornographiques.

Évidemment, il s'agit d'une pure provocation littéraire dont le côté parodique saute aux yeux et pour cette raison il faut prendre l'humour de Pybrac au second degré. C'est d'ailleurs dans l'absurdité qu'il faut chercher l'humour louÿsien. Celui-ci va de pair avec une ironie mordante grâce à laquelle l'écrivain peut aborder les sujets les plus piquants tout en évitant une éventuelle censure morale.

On retrouve cette même ironie dans le roman intitulé Trois filles de leur mère (1926), le livre le plus scandaleux ainsi que le plus insolent que Louÿs n'ait jamais écrit. L'écrivain y va jusqu'au cœur de l'obscène pour s'abandonner à la veine pornographique la plus effrénée, ne s'arrêtant devant aucun tabou. C'est bien grâce aux Trois filles de leur mère que nous pouvons mettre Louÿs à la hauteur de Sade ou de Bataille. Ses héroïnes s'y font mutuellement mal, se laissent brutalement sodomiser et abuser, rien ne leur déplaît, ni le sexe avec des animaux ni l'humiliation sous forme de perversions scatologiques. L'acte d'amour est ici inséparable de l'obscénité la plus radicale et baigne dans l'ambiance de la pire débauche et abjection. Or, en dépit de la cruauté et de l'obscénité des scènes du roman, nous sommes persuadée que le texte du roman ne fut pas écrit d'une plume sérieuse et que c'est une divertissante parodie érotique qu'il faut y voir.

L'absurdité des scènes est d'ailleurs évidente et le lecteur vigilant doit tout de suite remarquer une bonne dose d'humour louÿsien transparaissant même derrière les paroles les plus audacieuses. Par conséquent, on ne peut que sourire pendant les scènes (le caractère théâtral du roman est indéniable) telle que celle où Lilli, l'une des filles de la mère, se met à un exercice d'acrobatie devant le jeune homme pour arriver à se tordre suffisamment, afin de se lécher le vagin.

Finalement, le lecteur des Trois filles de leur mère finira par comprendre à quel point l'auteur du roman s'amusait à écrire une œuvre aussi provocatrice qu'absurde, se moquant des contemporains pudibonds. Dans le cas idéal, il s'amusera en même temps que lui.

Il est indéniable que la majorité écrasante de l'œuvre littéraire de Pierre Louÿs sent le soufre. Elle est subversive, scandaleuse, sulfureuse et insolite, certes, mais elle est également pleine d'humour. En effet, ce qui rend l'érotisme de Louÿs si singulier, c'est bien cette dose d'humour, de ce condiment indispensable sans lequel le texte ne serait pas le sien et grâce auquel toute son œuvre secrète est transfigurée. De ce fait, nous ne voyons qu'une seule voie interprétative possible de l'œuvre érotique de Louÿs - à travers le prisme de la parodie. Or, pour pouvoir 
y pénétrer et la comprendre, il faut avoir une disposition à l'humour bien particulière ainsi que la capacité à ne pas prendre les récits de Louÿs trop au sérieux.

Sans aucun doute, c'est une extraordinaire aptitude au pastiche et à la parodie et un sens aigu du comique de Louÿs qui donnent à l'écriture louÿsienne un caractère d'originalité en lui permettant de créer de véritables chefs-d'œuvre du genre.

\section{BIBLIOGRAPHIE}

Albert Nicole G. (2005), Saphisme et décadence dans Paris fin-de-siècle, Paris, Éditions de la Martinière.

ARON Paul (2009), Le pastiche comme objet d'étude littéraire. Quelques réflexions sur l'histoire du genre, Modèles linguistiques, $N^{o} 60$, p. 11-27.

Cogman Peter (2006), L'œuvre érotique de Pierre Louÿs comme exercice de style, in : D'ORMESSON Jean, PERRIER Jean-Claude (2002), Pierre Louÿs : ombres et lumières d'un poète libertin, Le Figaro, $N^{o}$ 17991, Le Figaro Littéraire, jeudi 13 juin 2002, p. 3-8.

DE MusseT Alfred (1888), Euvres complètes d'Alfred de Musset. Poésies, Paris, Charpentier.

Dousteyssier-KhOze Catherine ; Place-Verghnes Floriane (éd.), Poétiques de la parodie et du pastiche de 1850 à nos jours, Bern, Peter Lang, p. 95-106.

GouJON Jean-Paul (1988), La genèse de La femme et le pantin par Pierre Louÿs d'après des documents inédits, in : Estudios de lengua y literatura francesas. $N^{o}$ 2, Exemplaire dédicacé à: Literaturas de Fin de Siglo, Cadiz, Universidad de Cadiz, p. 71-84.

GoujON Jean-Paul (2012), Euvre érotique/Pierre Louÿs, Paris, Robert Laffont.

GouJON Jean-Paul (1998), Pierre Louÿs : du libertinage à l'érotomanie, Le Magazine littéraire, $N^{o} 371$, p. 51.

GouJON Jean-Paul (1991), Pierre Louÿs ou la subversion de la morale in: Numéro spécial sur la «Littérature d'une fin de siècle », Europe $N^{o}$ 751-752, p. 61-68.

GoujON Jean-Paul (2002), Pierre Louÿs : une vie secrète (1870-1925), Paris, Fayard.

JULLIAN Philippe (1969), Esthètes et magiciens, L'art fin de siècle, Paris, Librairie académique Perrin.

KYRIA Pierre (1991), Louÿs, érotomane et érudit, Le Magazine Littéraire, $N^{o} 288$, p. 52-53.

LE BRUn Annie (2000), De l'éperdu, Paris, Stock, 2000.

LOUŸS Pierre (1895), Les Chansons de Bilitis, poèmes en prose, Paris, L'Art indépendant.

MALAIS Nicolas (2006-2007), Pierre Louÿs, bibliophile, érotomane et amoureux, Le Frisson Esthétique, $N^{o} 3$, p. 64-69.

PAUVERT Jean-Jacques (1995), Anthologie historique des lectures érotiques de Sade à Victoria 1791-1904, Paris, Stock/Spengler. 
PAUVERT Jean-Jacques (2004), Préface in : LOUŸs Pierre, Manuel de Gomorrhe suivie de l'île aux dames, Paris, La Musardine.

PIA Pascal (1998), Les Livres de l'Enfer : bibliographie critique des ouvrages érotiques dans leurs différentes éditions du XVI siècle à nos jours, Paris, Fayard.

PIERRE José (1991), L’Univers symboliste, Fin de siècle et décadence, Paris, Éditions Aimery Somogy.

ŠTÁFOVÁ Vendula (2014), L'Éros décadent dans l'œuvre de Pierre Louÿs, thèse de doctorat, manuscrit, Brno, Université Masaryk de Brno. 